\title{
Safety and Efficacy of Trifluridine-Tipiracil Hydrochloride Oral Combination (TAS-102) in Patients with Unresectable Colorectal Cancer
}

\author{
SATOSHI NARIHIRO ${ }^{1}$, KATSUHITO SUWA ${ }^{1}$, TAKURO USHIGOME ${ }^{1}$, MASAMICHI OHTSU ${ }^{1}$, \\ SYUNJIN RYU ${ }^{1}$, YUYA SHIMOYAMA ${ }^{1}$, TOMOYOSHI OKAMOTO ${ }^{1}$ and KATSUHIKO YANAGA ${ }^{2}$ \\ ${ }^{1}$ Department of Surgery, The Jikei University Daisan Hospital, Tokyo, Japan; \\ ${ }^{2}$ Department of Surgery, The Jikei University School of Medicine, Tokyo, Japan
}

\begin{abstract}
Aim: To retrospectively examine efficacy and safety of oral combination of trifluridine and tipiracil hydrochloride (TAS-102) as the second-line therapeutic agent for unresectable colorectal cancer. Patient and Methods: Treatment outcomes of 17 patients who had received TAS-102 at our Institution from January 2015 to January 2017 were analyzed. The indications for second-line TAS-102 treatment were intolerance to other multi-drug combination (four patients) or patient refusal of the standard second-line therapy (13 patients). Results: Among 17 patients who received TAS-102 as second-line therapy, partial response was observed in two (12\%) and stable disease in two (12\%). Outcomes of TAS-102 given as second-line therapy were: median overall survival of 5 months, response rate of $12 \%$ and disease control of $24 \%$. Overall, no adverse events other than neutropenia were noted. Conclusion: Our findings suggest a beneficial role of TAS-102 in second-line therapy for unresectable colorectal carcinoma.
\end{abstract}

In recent years, pharmacological treatment of unresectable advanced and recurrent colorectal cancer has progressed extensively. Although many improvements and broader applications have been achieved, some patients cannot receive optimal therapies due to undesirable adverse effects or intolerance to anticancer medications. An oral antitumor drug, which is a combination of trifluridine and tipiracil

This article is freely accessible online.

Correspondence to: Satoshi Narihiro, MD, Department of Surgery, The Jikei University Daisan Hospital, 4-11-1 Izumihoncho, Komae city, Tokyo 201-8601, Japan. Tel: +81 334801151 (ext. 3251), Fax: +81 334808295, e-mail: nasa1355@yahoo.co.jp

Key Words: TAS-102, chemotherapy, unresectable colorectal carcinoma. hydrochloride (TAS-102) has been shown to be effective in patients with unresectable metastatic colorectal cancer refractory to standard chemotherapies. It was approved for use in Japan in March 2014 as a third-line chemotherapeutic $(1,2)$. Although usage in combination with other molecularly-targeted agents for second-line therapy has recently been reported (3), no studies have addressed safety and efficacy of TAS-102 applied as a single agent in second-line treatment. In the present study, we examined the efficacy and safety of TAS-102 given as second-line therapy for patients for whom the firstline multi-drug treatment including adjuvant chemotherapy could not be continued or second-standard chemotherapy options were not chosen by patients themselves.

\section{Patients and Methods}

With approval of the Institutional Review Board (protocol 27283[8168]), 17 patients who received TAS-102 as second-line therapy between January 2015 and January 2017 at The Jikei University School of Medicine Daisan Hospital were enrolled. According to RECIST 1.1 criteria (4), response rate, disease control rate and adverse effects were retrospectively examined. All patients had been diagnosed with unresectable metastatic colorectal cancer, and comparative parameters consisted of: sex, age, performance status (PS), primary tumor site, resected/not resected primary tumor, treatment stage, number of TAS-102 doses, organs of metastasis and number of metastases. TAS- 102 was given orally at $35 \mathrm{mg} / \mathrm{m} 2$ twice a day for 28 days (one course): 2-Week cycles of 5 days of treatment and 2 days of rest, followed by 14 days of rest. All data analyses were performed using Microsoft Excel software.

\section{Results}

Seventeen patients received TAS-102 during the study period. The median age of patients was 65 years (range $=37-$ 83 years) and all had PS of 1 or 2; the majority of patients were men. The colon was the most frequent primary site and in more than half of the patients, the primary lesion had been resected. Previous chemotherapies included: One regimen in 
four patients and more than two regimens in the remaining 13 patients. Thirteen patients received fewer than 10 courses of chemotherapy and four patients completed 10 or more courses of TAS-102-containing chemotherapy, respectively (Table I). For all patients who received TAS-102 as secondline therapy, TAS-102 was selected as the sole antitumor agent and patients had been instructed that TAS-102 was not the standard treatment option. Informed consent to TAS-102 use was obtained from all patients before starting therapy.

Four out of the 17 patients received TAS-102 alone as second-line therapy, for whom the reasons for TAS-102 selection consisted of patient's request in two, severe adverse effects (severe hepatoxicity) of first-line adjuvant chemotherapy in one, and ineffective 5-fluorouracil containing postoperative adjuvant chemotherapy in one patient, respectively (Table II).

The patient who developed severe hepatotoxicity had first received 12 courses of adjuvant chemotherapy [folinic acid, 5-fluorouracil and oxaliplatin (FOLFOX) regimen], then developed liver metastases and was additionally given six courses of folinic acid, 5-fluorouracil and irinotecan (FOLFIRI) plus an anti- epidermal growth factor agent (EGFR). For this patient, TAS-102 was selected as secondline treatment because of expected severe hepatoxicity due to FOLFOX/FOLFIRI treatment.

The patients whose disease was refractory to 5-FU had initially received TS-1 as the postoperative adjuvant chemotherapy agent, then developed liver metastases and was additionally given four courses of FOLFIRI plus anti-EGFR agent. Following both therapies, the patient developed grade 3 diarrhea and the diagnosis of 5-FU intolerance was made.

Outcome analyses revealed partial response (PR) in two patients (12\%), stable disease (SD) in two (12\%), progressive disease (PD) in six (35\%), and unevaluable treatment results in seven patients $(41 \%)$ (Table III). All patients with either PR or SD had received TAS-102 as second-line therapy.

Amongst all patients enrolled, the overall response rate to TAS-102 therapy (PR or above) was, therefore, 12\%, and sufficient disease control (SD or above) was achieved in $24 \%$. When the four patients given TAS-102 as second-line treatment were separately analyzed, response and disease control rates were $50 \%$ and $100 \%$, respectively. Considering all 17 patients, median overall survival (OS) was 5 months (range $=1-24$ months) and while for the four patients who completed 10 or more courses of TAS-102 as second-line therapy, the survival was 15 months (range $=12-24$ months) (Table IV).

Among all patients treated with TAS-102, neutropenia developed in 11 patients (grade 1 in two, grade 2 in four and grade 3 in five patients), and no other adverse events were encountered recorded (Table V). In some patients, the rest period had to be extended due to side-effects but for none of the patients was treatment terminated due to adverse effects.
Table I. Demographics of all study patients.

\begin{tabular}{lc}
\hline Parameter & Value \\
\hline Gender: M:F & $12: 5$ \\
Median age: range, years & $65(35-83)$ \\
Performance status: $1: 2$ & $10: 7$ \\
Primary tumor site: colon:rectum & $12: 5$ \\
Resection of primary tumor: Performed:not performed & $14: 3$ \\
Number of metastases: $1: 2: 3$ & $7: 9: 1$ \\
Organ with metastasis: Liver:lungs: & \\
peritoneum:lymph nodes:other & $6: 10: 3: 4: 4$ \\
Number of previous chemotherapeutic regimens: $1: 2: 3: 4$ & $4: 5: 6: 2 \dagger$ \\
Number of therapy courses: $<10: \geq 10$ & $13: 4$ \\
\hline
\end{tabular}

M: Male, F: female; †overlapping values.

\section{Discussion}

In 2012, OS of patients with unresectable metastatic colorectal carcinoma receiving TAS-102 versus placebo was studied by a double-blind, randomized (2:1 ratio, treatment $v s$. placebo), controlled phase II trial performed in Japan (J003 trial) (1). The study findings demonstrated longer median OS in the group treated with TAS-102, which was then approved for use in Japan in March, 2014. Results of the RECOURSE study of patients with refractory metastatic colorectal cancer demonstrated that patients treated with TAS-102 had longer OS compared to those treated with placebo (7.1 vs. 5.3 months) as well as longer progressionfree survival (2). Based on the findings from such study results, one might consider that the role and position of TAS102 in salvage therapy should be similar to those by regorafenib; however, when compared to regorafenib, TAS102 produces less non-hematological side-effects $(5,6)$. Thus, the safety profile makes TAS-102 a reasonable candidate for salvage therapy and underlines recent wider use of TAS-102 in second-line treatments combined with molecularly targeted anticancer drugs $(3,7)$.

In the current study, the efficacy of TAS-102, when assessed according to RECIST criteria, was more or less satisfactory, with median OS of 5 months, response rate of $12 \%$ and disease control rate of $24 \%$. In terms of response rate, the current study demonstrated even better outcome as compared to both J003 and RECOURSE trials. All four patients with response rate of $\mathrm{PR}$ or SD according to the RECIST criteria, had received TAS-102 as second-line therapy; they exhibited a good response rate of $50 \%$ and disease control rate of $100 \%$, and median OS in such patients was 15 months, which was generally not inferior to OS of the standard treatments: 12.9 months with FOLFOX plus bevacizumab (8), 13.3 months with FOLFIRI plus bevacizumab (9), 14.53 months with 
Table II. Demographics of patients who received 10 or more courses of TAS-102 as sole second-line therapy.

\begin{tabular}{lcccccc}
\hline Gender & $\begin{array}{c}\text { Age } \\
\text { (years) }\end{array}$ & PS & $\begin{array}{c}\text { Number of } \\
\text { courses }\end{array}$ & RECIST & $\begin{array}{c}\text { Treatment period, } \\
\text { months }\end{array}$ & $\begin{array}{c}\text { Reason for TAS-102 } \\
\text { as second-line treatment }\end{array}$ \\
\hline $\mathrm{M}$ & 63 & 2 & 15 & PR & 18 & Patient's request \\
$\mathrm{M}$ & 60 & 1 & 10 & PR & 12 & Severe hepatotoxicity \\
$\mathrm{M}$ & 67 & 1 & 18 & SD & 24 & 5-Fluorouracil intolerance \\
$\mathrm{F}$ & 75 & 1 & 10 & SD & 12 & Patient's request \\
\hline
\end{tabular}

M: Male, F: female; PS: performance status; RECIST: Response Evaluation Criteria in Solid Tumors; PR: partial response; SD: stable disease.

FOLFIRI plus panitumumab (10), and 18 months with irinotecan plus S-1 plus bevacizumab (11). All four patients in the current study received more than 10 courses for over 12 months on average, which suggests that a longer administration period might be required in order to observe the effects of TAS-102 and might also suggest that an early start and long continuation of the regimen may achieve longer OS, higher response rates, and better disease control rates.

Frequent and significant hematotoxicity caused by TAS102 are well-known (2), but non-hematological toxicity has been observed to be rather weak (12). Febrile neutropenia occurred in $3.8 \%$ and $4.4 \%$ of TAS-102-treated patients in the RECOURSE (2) and J003 (1) trials, respectively. Among the 17 patients in our study, neutropenia developed in 11 patients $(64 \%)$, but no other adverse events were recorded. Furthermore, none of the patients receiving TAS-102 needed to have therapy terminated due to adverse events and all patients completed the therapy, however, we did find the need to individually adjust rest periods. The results of the current study do not indicate that the position of TAS-102 within the available chemotherapy choices should change; in Japanese colon cancer treatment guidelines (13) and NCCN guidelines (14) TAS-102 remains a the third- line (or above) therapy.

Generally, while longer OS or progression-free survival have been observed after TAS-102 therapy $(1,2)$, the response rate in patients receiving the drug has not improved remarkably. Such observations suggest that there are still many unresolved issues regarding appropriate dosages, treatment periods or regimens for TAS-102 therapy. Many studies are still required to establish the most effective TAS102-based regimens (15-20).

The results of the current study suggest potential advantageous effects of early administration and long continuation of TAS-102 therapy provided appropriate rest periods between therapy courses are individually adjusted. Thus, with the lowest possible incidence of TAS-102 therapy-related adverse events, the drug might be safely applied and tolerated in patients with advanced recurrent
Table III. Outcomes of second-line TAS-102 therapy according to Response Evaluation Criteria in Solid Tumors.

\begin{tabular}{lc}
\hline & Number of patients (\% out of 17) \\
\hline PR & $2(12 \%)$ \\
SD & $2(12 \%)$ \\
PD & $6(35 \%)$ \\
Unevaluable & $7(41 \%)$ \\
\hline
\end{tabular}

PR: Partial response; SD: stable disease; PD: progressive disease.

Table IV. Outcomes of second-line TAS-102 therapy.

\begin{tabular}{lcc}
\hline & $\begin{array}{c}\text { All patients } \\
(\mathrm{n}=17)\end{array}$ & $\begin{array}{c}\geq 10 \text { courses of } \\
\text { TAS-102 }(\mathrm{n}=4)\end{array}$ \\
\hline Median overall survival (range), months & $5(1-24)$ & $15(12-24)$ \\
Response rate & $12 \%$ & $50 \%$ \\
Disease control rate & $24 \%$ & $100 \%$ \\
\hline
\end{tabular}

Table V. Degree of neutropenia.

\begin{tabular}{lc}
\hline Grade & Number of patients (\%) \\
\hline 1 & $2(18 \%)$ \\
2 & $4(36 \%)$ \\
3 & $5(45 \%)$ \\
Total & 11 \\
\hline
\end{tabular}

colorectal cancer refractory to or not tolerating other chemotherapies. In conclusion, side-effects of TAS-102 as single-agent therapy in patients with unresectable colorectal cancer who had undergone first-or second-line chemotherapy is safe and for selected patients, the use of TAS-102 as a second-line chemotherapy could be satisfactory. 


\section{Disclosure}

All Authors have no conflict of interest in regard to this study.

\section{References}

1 Yoshino T, Mizunuma N, Yamazaki K, Nishina T, Komatsu Y, Baba H, Tsuji A, Yamaguchi K, Muro K, Sugimoto N, Tsuji Y, Moriwaki T, Esaki T, Hamada C, Tanase T and Ohtsu A: TAS102 monotherapy for pretreated metastatic colorectal cancer: a double-blind, randomized, placebo-controlled phase 2 trial. Lancet Oncol 10: 993-1001, 2012.

2 Mayer RJ, Van Custen E, Falcone A, Yoshino T, Garcia-Carbonero R, Mizunuma N, Yamazaki K, Shimada Y, Taberneo J, Komatsu Y, Sobrero A, Boucher E, Peeters M, Zaniboni A, Hochster H, Cleary JM, Prenen H, Benedetti F, Mizuguchi H, Makris L, Ito M and Ohtsu A: Randomized trial of TAS-102 for refractory metastatic colorectal cancer. N Eng J Med 372: 1909-1919, 2015.

3 Tsukihara H, Nakagawa F, Sakamoto K, Isida K, Tanaka N, Okabe H, Uchida J, Matsuo K and Takechi T: Efficacy of combination chemotherapy using a novel oral chemotherapeutic agent, TAS-102, together with bevacizumab, cetuximab, or panitumumab on human colorectal cancer xenografts. Oncol Rep 33: 2135-2142, 2015.

4 Eisenhauer EA, Therasse P, Bogaerts Jet, Schwartz LH, Sargent D, Ford R, Dancey J, Arbuck S, Gwyther S, Mooney M, Rubinstein L, Shankar L, Dodd L, Kaplan R, Lacombe D and Verweij J: New Response Evaluation Criteria in solid Tumours: Revised RECIST guideline (version 1.1). Eur J Cancer 45: 228-247, 2009.

5 Grothey A, Van Cutsem E and Sobrero A: Regorafenib monotherapy for previously treated metastatic colorectal cancer (CORERCT): Lancet 381(9863): 303-312, 2013.

6 Kotani D, Shitara K, Kawazoe A, Fukuoka S, Kuboki Y, Bando H, Okamoto W, Kojima T, Doi T, Ohtsu A and Yoshino T: Safety and efficacy of trifluridine/tipiracil monotherapy in clinical practice for patients with metastatic colorectal cancer: experience at a single institution. Clinical Colorectal Cancer 15: 109-115, 2016.

7 Kuboki Y, Nishina T, Shinozaki E, Yamazaki K, Shitara K, Okamoto W, Kajiwara T, Matsumoto T, Tsushima T, Mochizuki N, Nomura S, Doi A, Sato A, Ohtu A and Yoshino T: TAS-102 plus bevacizumab for patients with metastatic colorectal cancer refractory to standard therapies (C-TASK FORCE): an investigator-initiated, open-label, single-arm, multicenter, phase1/2 study. Lancet Oncol 17: 1470-2045, 2017.

8 Giantonio BJ, Catalano PJ, Meropol NJ, O'Dwyer PJ, Mitchell EP, Alberts SR and Schwartz MA: Bevacizumab in combination with oxaliplatin, fluorouracil, and leucovorin (FOLFOX4) for previously treated metastatic colorectal cancer: results from the Eastern Cooperative Oncology Group Study E3200. J Clin Oncol 25: 1539-1544, 2007.

9 Tabernero J, Yoshino T, Cohn AL, Obermannova R, Bodoky G, Garcia-Carbonero R, Ciuleanu TE, Portnoy DC, Van Cutsem E, Grothey A, Prausová J, Garcia-Alfonso P, Yamazaki K, Clingan PR, Lonardi S, Kim TW, Simms L, Chang SC and Nasroulah F: Ramucirumab versus placebo in combination with second-line FOLFIRI in patients with metastatic colorectal carcinoma that progressed during or after first-line therapy with bevacizumab, oxaliplatin, and a fluoropyrimidine (RAISE): A randomised, doubleblind, multicentre, phase 3 study. Lancet Oncol 16: 499-508, 2015.

10 Peeters M, Price TJ and Cervantes A: Randomized phase III study of panitumumab with fluorouracil, leucovorin, and irinotecan
(FOLFIRI) compared with FOLFIRI alone as second-line treatment in patients with metastatic colorectal cancer. Acta Oncol 51: 867-72, 2012.

11 Komatsu Y, Yuki S and Sogabe S: Phase II study of combined chemotherapy with irinotecan and S-1 (IRIS) plus bevacizumab in patients with inoperable recurrent or advanced colorectal cancer. Acta Oncol 51: 867-872, 2012.

12 Yoshimatsu K, Yokomizo H and Nakayama M: Short-term outcome of TAS-102 for refractory metastatic colorectal cancer. Jpn J Cancer Chemother 42: 1316-1318, 2015.

13 Watanabe T, Muro K, Ajioka Y, Hashiguchi Y, Ito Y, Saito T, Hamaguchi T, Isida H, Ishiguro M, Ishihara S, Kanemitsu Y, Kawano H, Kinugasa Y, Kokudo N, Murofushi K, Nakajima T, Oka S, Sakai Y, Tsuji A, Uehara K, Ueno H, Yamazaki K, Yoshida M, Yoshino T, Boku N, Fujimori T, Itabashi M, Koinuma N, Morita T, Nishimura G, Sakata Y, Shimada Y, Takahashi K, Tanaka S, Tsuruta O, Yamaguchi T, Yamaguchi N, Tanaka T, Kotake K and Sugihara K: Japanese Society for Cancer of the Colon and Rectum (JSCCR) guidelines 2016 for the treatment of colorectal cancer. Int J clin Oncol 23: 1-34, 2018.

14 Benson AB 3rd, Venook AP, Cederquist L, Chan E, Chen YJ, Cooper HS, Deming D, Engstrom PF, Enzinger PC, Fichera A, Grem JL, Grothey A, Hochster HS, Hoffe S, Hunt S, Kamel A, Kirilcuk N, Krishnamurthi S, Messersmith WA, Mulcahy MF, Murphy JD, Nurkin S, Saltz L, Sharma S, Shibata D, Skibber JM, Sofocleous CT, Stoffel EM, Stotsky-Himelfarb E, Willett CG, Wu CS, Gregory KM and Freedman-Cass D: Colon Cancer, Version 1.2017, NCCN Clinical Practice Guidelines in Oncology. J Natl Compr Canc Netw 15: 370-398, 2017.

15 Arita S, Shirakawa T, Matsushita Y, Shimokawa HK, Hirano G, Makiyama A, Shibata Y, Tamura S, Esaki T, Mitsugi K, Ariyama $\mathrm{H}$, Kusaba H, Akashi K and Baba E: Efficacy and safety of TAS102 in clinical practice of salvage chemotherapy for metastatic colorectal cancer. Anticancer Res 36: 1959-1966, 2016.

16 Kawahara H, Mouri T, Ishida K, Matsumoto N, Akiba T and Yanaga K: Usefulness of TAS-102 as third-line chemotherapy for metastatic colorectal cancer. Anticancer Res 38: 2419-2422, 2018.

17 Jiezhong C, Mei H and Muhammad WS: TAS-102 an emerging oral fluoropyrimidine. Anticancer Res 36: 21-26, 2016.

18 Suenaga M, Schirripa M, Cao S, Zhang W, Yang D, Dadduzio V, Salvatore L, Borelli B, Pietantonio F, Ning Y, Okazaki S, Berger MD, Miyamoto Y, Gopez Jr., Barzi A, Yamaguchi T, Loupakis F and Lenz HJ: Potential role of polymorphisms in the transporter genes ENTI and MATE1/OCT2 in predicting TAS-102 efficacy and toxicity in patients with refractory metastatic colorectal cancer. European Journal of Cancer 86: 197-206, 2017.

19 Yasue F, Kimura M, Usami E, Iwai M, Go M, Kawachi S, Mitsuoka M, Ikeda Y and Yoshimura T: Risk factors contributing to the development of neutropenia in patients receiving oral trifluridine-tipiracil (TAS-102) chemotherapy for advanced/ recurrent colorectal cancer. Pharmazie 73: 178-181, 2018.

20 Yoshida Y, Sakamoto R, Kajitani R, Munechika T, Matsumoto Y, Komono A, Aisu N, Daibo K, Kiyomi F and Hasegawa S: Biweekly administration of TAS-102 for neutropenia prevention in patients with colorectal cancer. Anticancer Res 38: 4367-4373, 2018.

Received August 1, 2018 Revised September 14, 2018 Accepted September 19, 2018 